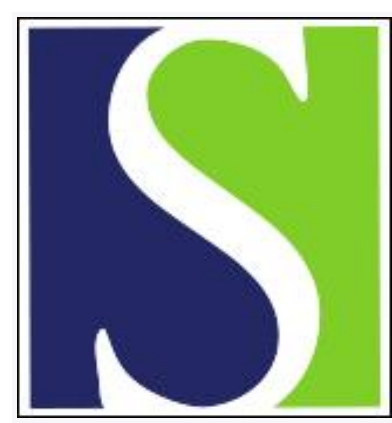

Scand J Work Environ Health 1992;18(4):242-245

https://doi.org/10.5271/sjweh.1583

Issue date: 01 Aug 1992

An apparently incongruous exposure-response relationship resulting from the use of job description to assess magnetic field exposure.

by Delpizzo V

Affiliation: Australian Radiation Laboratory, Yallambie.

This article in PubMed: www.ncbi.nlm.nih.gov/pubmed/1411366

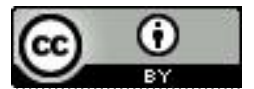




\title{
An apparently incongruous exposure-response relationship resulting from the use of job description to assess magnetic field exposure
}

\author{
by Vincent Delpizzo, PhD'
}

\begin{abstract}
DELPIZZO V. An apparently incongruous exposure-response relationship resulting from the use of job description to assess magnetic field exposure. Scand J Work Environ Health 1992;18:242-5. Empirical data are presented to support the observation that nondifferential misclassification of subjects classified according to an ordinal scale can result in an artifactual nonmonotonic exposure-response curve. This phenomenon can be interpreted erroneously as an indication that an observed relationship is not causal. The occupational exposure of 40 subjects to extremely low-frequency magnetic fields was estimated on the basis of their job description while their "true" exposure was determined by direct dosimetry. A "true" risk was assumed to exist, and estimates of risk that could be obtained through hypothetical case-referent or cohort studies were calculated.
\end{abstract}

Key terms: causality, epidemiology, extremely low frequency, misclassification, surrogates, trend.

A monotonic relationship of increasing risk for increasing exposure to an agent is normally regarded as one criterion in favor of interpreting an association as causal. In some cases, the risk for the group with the highest exposure classification is found to be lower than that observed for an intermediate exposure class, and this fact is taken as an indication that some artifact may be responsible for the association. For example, McDonald et al (1) reported that the ratio of observed-to-expected spontaneous abortions among women using video display units for $1-6 \mathrm{~h}$ a week was 1.24 , for those using such units for $7-29 \mathrm{~h}$ a week it was 1.25 , while it was only 1.12 for those using such a unit for $\geq 30 \mathrm{~h}$ week. This finding prompted Bryant \& Lowe (2) to comment that "this lack of a doseresponse relationship makes a causal relationship difficult to defend [p 133]."' Keam (3) used "anomalous wire coding results [p 86]" [ie, unexpectedly high odds ratio (OR) estimates for next-to-lowest residence classification] as an argument to reject as causal the association between residence in proximity to power lines and increased cancer risk. Tomenius et al (4) found a significant association between childhood cancer and residence in homes where elevated magnetic fields were measured. Under particular definitions of exposure, their data also show an inconsistent exposure-response relationship. For example, if low exposure is defined as a measured magnetic field of $<3 \mathrm{mG}$ and high exposure as a field of $\geq 6 \mathrm{mG}$, one obtains an OR of 2.27 for medium exposure and 1.74 for high exposure.

1 Australian Radiation Laboratory, Yallambie, Australia.

Reprint requests to: Dr V Delpizzo, Australian Radiation Laboratory, Lower Plenty Road, Yallambie, Victoria 3085, Australia.
Dosemici et al (5) pointed out that, under some nondifferential misclassification conditions, a false inverse trend may be created for the exposure-response relationship. They acknowledged that the misclassification patterns presented in their hypothetical examples were extreme.

This paper aims to point out that the conditions required to create such a strong distortion are not unrealistic. This observation is supported by experimental data pertaining to the issue of classification of subjects occupationally exposed to extremely low-frequency (ELF) magnetic fields.

Misclassification can act in the following two ways to reverse the trend of the estimated relative risk (RR) as a function of exposure: (i) it affects stronger associations proportionally more than weak ones [see, for example, Flegal et al (6) and Gladen \& Rogan (7)] and (ii) it can artifactually increase the estimate of risk (5), provided that a true risk exists (ie, nondifferential misclassification cannot artifactually create an association).

The latter observation runs against the commonly held view that nondifferential misclassification can only bias the estimate of risk towards the no-effect value, and therefore it may merit a brief explanation. Although nondifferential misclassification always biases the estimate of risk towards unity in the case of a dichotomous analysis, this is not necessarily the case if subjects are classified according to an ordinal exposure scale. Consider, for example, a study using a three-level (ie, high, medium, and low) exposure scale and assume that all low-exposure subjects are correctly classified, whereas there is misclassification between the medium- and high-exposure classes. In this case, provided that there is a true risk associated with exposure, the population in the high-exposure class will 
contain fewer subjects at risk than would have been the case in the absence of misclassification, and the risk relative to the low-exposure population will appear artificially low. However, the same misclassification has artificially increased the number of subjects at risk in the medium-exposure group, and the corresponding relative risk will appear spuriously higher. This situation is not infrequently encountered in practice. For example, it is easier to classify nondrinkers or nonsmokers as unexposed than to correctly classify "moderate" or "heavy" smokers or drinkers.

In the course of a study of the use of personal dosimeters to determine occupational exposure to ELF magnetic fields, some data were obtained that offer a quantitative example of this occurrence.

\section{Materials and methods}

Some of the staff of the Australian Radiation Laboratory were classified with respect to magnetic field exposure on the basis of their work description. Office workers were regarded as "low-exposure" subjects, and mechanical workshop staff and electronic technicians were classified as "medium-" and "high-exposure," respectively. This intuitive classification was supported by a limited number of "spot" magnetic field measurements that also suggested exposure "scores" for the three classes. Office workers appeared to be typically exposed to $<1 \mathrm{mG}$ and were assigned an exposure score of zero, mechanical workshop staff to $1-2 \mathrm{mG}$ (exposure score $=1$ ), and electronic technicians to $\geq 2 \mathrm{mG}$ (exposure score $=2$ ).

The surrogate having thus been defined and validated, several volunteers from each group were asked to wear six magnetic field dosimeters (AMEX-3D, marketed by Enertech Consultants, Campbell, California, United States) for a period of approximately $2 \mathrm{~h}$, while actually engaged in their typical occupational activities. Direct broadband $(35-1000 \mathrm{~Hz})$ magnetic field measurements were thus obtained at the head, the chest, the abdomen, the right hip, the right knee, and the right ankle. The average magnetic field thus recorded was assumed to be the "true" exposure.

\section{Results}

The data collected are summarized in figure 1 and table 1 . On the basis of these data, job description appears to be an acceptable surrogate for dichotomous analysis (for "exposure" defined as a whole-bodyaveraged magnetic field of $\geq 1 \mathrm{mG}$, it had a sensitivity of 0.8 and a specificity of 0.9 ). It also had some potential for use in ordinal scale analysis since the median values for each exposure class were in ascending order. However, the exposure score assigned to the high-exposure class appeared to be too high. In addition, although low-exposure subjects were classified with relatively good accuracy, there was a high degree

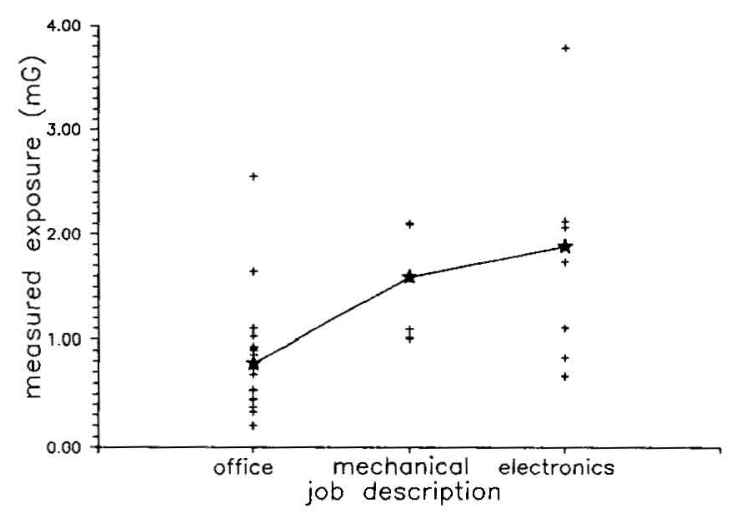

Figure 1. Scatter plot of the measured, whole-body-averaged magnetic flux density exposure and the job description classification. The stars represent the median of the magnetic fields measured for each job grouping. The highest data points for both the medium- and high-exposure classifications lie outside the plot area.

Table 1. Classification of occupational exposure to magnetic fields by direct dosimetry and job description. ( $N=$ number of subjects)

\begin{tabular}{lcccc}
\hline \multirow{2}{*}{$\begin{array}{l}\text { Classification } \\
\text { by surrogate } \\
\text { (job description) }\end{array}$} & $\begin{array}{c}\text { "True" classification } \\
\text { (body-worn dosimeters) }\end{array}$ \\
\cline { 2 - 5 } & $\begin{array}{c}\text { High } \\
(\mathrm{N})\end{array}$ & $\begin{array}{c}\text { Medium } \\
(\mathrm{N})\end{array}$ & $\begin{array}{c}\text { Low } \\
(\mathrm{N})\end{array}$ & $\begin{array}{c}\text { Total } \\
(\mathrm{N})\end{array}$ \\
\hline High & 4 & 3 & 2 & 9 \\
Medium & 4 & 5 & 0 & 9 \\
Low & 1 & 3 & 18 & 22 \\
\hline Total & 9 & 11 & 20 & 40 \\
\hline
\end{tabular}

of reciprocal misclassification between the high- and medium-exposure classes. These factors would have serious effects on the estimate of the exposure-response relationship, as well as on the estimates of relative risk.

To estimate these effects, assume that the association between suspected occupational exposure to magnetic fields and increased cancer risk reported by several people (see, for example, references 8,9 , and 10 for reviews of the pertinent literature) is truly causal.

Assume an increase in relative risk of 0.4 per milligauss of exposure. (This value is arbitrary, but of the order of magnitude of the reported relative risks.)

Although very few data exist on occupational exposure to ELF magnetic fields, it is realistic to assume a prevalence of exposure skewed to the right (ie, fewer subjects exposed to high fields than to low fields).

On the basis of these hypotheses, we can postulate that a case-referent study of 1000 cases and 1000 referents would, if the subjects were correctly classified, exhibit the following population profile:

\section{exposure}

low $(0.00-0.99 \mathrm{mG})$ medium $(1.00-1.99 \mathrm{mG})$

high $(\geq 2 \mathrm{mG})$ cases controls odds ratio

$\begin{array}{lll}391 & 500 & 1.00 \\ 328 & 300 & 1.40 \\ 281 & 200 & 1.80\end{array}$


Table 2. Cancer risk estimates obtained from hypothetical cohorts exhibiting a pattern of misclassification similar to that found for the 40-subject sample. (MF = magnetic field, $\mathrm{RR}=$ relative risk)

\begin{tabular}{|c|c|c|c|c|c|c|c|c|}
\hline \multirow{3}{*}{ Exposure } & \multicolumn{4}{|c|}{ A Classification by surrogate } & \multicolumn{4}{|c|}{$\begin{array}{c}\text { B "True" classification (based on } \\
\text { personal dosimetry) }\end{array}$} \\
\hline & \multicolumn{2}{|c|}{ MF level (mG) } & \multirow{2}{*}{$\begin{array}{l}\text { Average } \\
\text { cancer } \\
\text { risk }\left(\overline{\mathrm{R}}_{\mathrm{j}}\right)\end{array}$} & \multirow{2}{*}{$\begin{array}{l}\text { Apparent } \\
\text { RR }\end{array}$} & \multicolumn{2}{|c|}{ Measured MF level (mG) } & \multirow{2}{*}{$\begin{array}{l}\text { Average } \\
\text { cancer } \\
\text { risk }\left(\overline{\mathrm{R}}_{\mathrm{j}}\right)\end{array}$} & \multirow{2}{*}{$\begin{array}{l}\text { "True" } \\
\text { RR }\end{array}$} \\
\hline & Nominal & True & & & Range & Mean & & \\
\hline $\begin{array}{l}\text { Low } \\
\text { (office workers) }\end{array}$ & $0.00-0.99$ & 0.83 & 1.33 & 1.00 & $0.00-0.99$ & 0.66 & 1.26 & 1.00 \\
\hline $\begin{array}{l}\text { Medium } \\
\text { (mechanical workshop } \\
\text { staff) }\end{array}$ & $1.00-1.99$ & 6.95 & 3.78 & 2.84 & $1.00-1.99$ & 1.39 & 1.56 & 1.24 \\
\hline $\begin{array}{l}\text { High } \\
\text { (electronics } \\
\text { technicians) }\end{array}$ & $\geq 2.00$ & 2.35 & 1.94 & 1.46 & $\geq 2.00$ & 8.14 & 4.26 & 3.38 \\
\hline
\end{tabular}

From table 1, the following misclassification probabilities can then be obtained: $P_{12}=0.00, P_{13}=0.10$, $P_{21}=0.27, P_{23}=0.27, P_{31}=0.11$, and $P_{32}=0.44$, where $P_{i j}$ is the fraction of subjects truly in exposure level $\mathrm{i}$ misclassified in exposure class $\mathrm{j}(1=$ low, $2=$ medium, 3 = high).

The population profiles that can be expected to be observed if the subjects are empirically classified on the basis of job description are obtained from the correctly classified population according to the following equations $(11,12)$ :

$$
\begin{aligned}
\hat{\mathrm{M}}_{3}= & \mathrm{M}_{3}-\mathrm{M}_{3} \cdot \mathrm{P}_{31}-\mathrm{M}_{3} \cdot \mathrm{P}_{32}+\mathrm{M}_{2} \\
& \cdot \mathrm{P}_{23}+\mathrm{M}_{1} \cdot \mathrm{P}_{13}, \\
\hat{\mathrm{M}}_{2}= & \mathrm{M}_{2}-\mathrm{M}_{2} \cdot \mathrm{P}_{23}-\mathrm{M}_{2} \cdot \mathrm{P}_{2 \mathrm{i}}+\mathrm{M}_{3} \\
& \cdot \mathrm{P}_{32}+\mathrm{M}_{1} \cdot \mathrm{P}_{12}, \\
\hat{\mathrm{M}}_{1}= & \mathrm{M}_{1}-\mathrm{M}_{1} \cdot \mathrm{P}_{13}-\mathrm{M}_{1} \cdot \mathrm{P}_{12}+\mathrm{M}_{3} \\
& \cdot \mathrm{P}_{31}+\mathrm{M}_{2} \cdot \mathrm{P}_{21}
\end{aligned}
$$

(equation 1a)

(equation 1b)

(equation 1c)

and

$$
\begin{aligned}
\hat{\mathrm{N}}_{3}= & \mathrm{N}_{3}-\mathrm{N}_{3} \cdot P_{31}-\mathrm{N}_{3} \cdot \mathrm{P}_{32}+\mathrm{N}_{2} \\
& \cdot \mathrm{P}_{23}+\mathrm{N}_{1} \cdot \mathrm{P}_{13}, \\
\hat{\mathrm{N}}_{2}= & \mathrm{N}_{2}-\mathrm{N}_{2} \cdot \mathrm{P}_{23}-\mathrm{N}_{2} \cdot \mathrm{P}_{21}+\mathrm{N}_{3} \\
& \cdot \mathrm{P}_{23}+\mathrm{N}_{1} \cdot \mathrm{P}_{12}, \\
\hat{\mathrm{N}}_{1}= & \mathrm{N}_{1}-\mathrm{N}_{1} \cdot \mathrm{P}_{13}-\mathrm{N}_{1} \cdot \mathrm{P}_{12}+\mathrm{N}_{3} \\
& \cdot \mathrm{P}_{31}+\mathrm{N}_{2} \cdot \mathrm{P}_{21},
\end{aligned}
$$

(equation 2a)

(equation $2 b$ )

(equation 2c)

where $M_{i}$ and $N_{i}$ are the numbers of correctly classified cases and referents, respectively, in each exposure class and $\hat{\mathrm{M}}_{\mathrm{i}}$ and $\hat{\mathrm{N}}_{\mathrm{i}}$ are the corresponding numbers of empirically classified subjects.

Thus as a result of misclassification the above population profile is altered into the following:

\section{exposure}

low (office workers)

medium (mechanical

workshop)

high (electronics technicians) cases referents odds ratio

$471 \quad 553 \quad 1.00$

$275 \quad 226 \quad 1.43$

$254 \quad 221 \quad 1.35$

The odds ratio associated with the medium-exposure category is artificially inflated, and it appears to be higher than that associated with the high exposure class.

A limitation of this and similar methods is that they use misclassification rates and relative risks averaged over each individual cell. It is possible that most misclassification involves subjects whose true exposure is close to the boundary values between the different classes. If, as it happens in many (but not all) cases, risk is a continuous function of exposure, rather than of the exposure level, the subjects the most likely to be misclassified have similar risk factors. In this case, it is possible that the effects of misclassification may be overestimated. In fact, recent work $(13,14)$ shows the result may even be differential misclassification.

Given the hypothesized linear relationship, another simple approach can be used. The risk $R_{i}$ for exposed subject $i$ compared with that for an equivalent unexposed subject is:

$$
R_{i}=\left(1+\mathrm{mfd}_{\mathrm{i}} \cdot \mathrm{r}\right) \text {. }
$$

(equation 3)

Once the subjects have been grouped into exposure categories, the average cancer risk $\overline{\mathbf{R}}_{\mathbf{j}}$ for subjects in exposure category $\mathrm{j}$ is given by:

$$
\begin{aligned}
\bar{R}_{j} & =\left(\Sigma_{i} R_{i}\right)_{j} / n_{j}=\left[\Sigma_{i}\left(1+m f d_{i} \cdot r\right)\right]_{j} / n_{j} \\
& =\left(n_{j}+r \cdot \Sigma_{i} \operatorname{mfd}_{i}\right)_{j} / n_{j}=1+\operatorname{mfd}_{j} \cdot r,
\end{aligned}
$$

(equation 4)

where $n_{j}$ is the number of subjects in that category and $\mathrm{mfd}_{j}$ is the average magnetic field for those subjects. The relative risk between categories $j$ and $k$ is given by $\overline{\mathbf{R}}_{\mathrm{j}} / \overline{\mathbf{R}}_{\mathrm{k}}$.

This procedure was carried out for the classification based on surrogate assessment and the classification based on measured values. The relative risks obtained in the two cases are shown in table 2 . Since most electronics technicians receive exposure higher than that received by most mechanical workshop staff, the relative risk trend in table $2 \mathrm{~A}$ appears paradoxical. However, because of the high (and in one case very high) exposure of some mechanical workshop workers and 
the rather low exposure of several electronic technicians, the average magnetic field exposure (and therefore the average risk) for the former group was higher than that for the latter.

\section{Discussion}

The presented data, although limited, confirm that nondifferential misclassification (ie, independent of the end point) may in practice have a magnitude and direction such that a linear, true exposure-response relationship may be distorted so as to appear inconsistent with causation.

Both the numerical and the analytical methods used indicated that classification based on job description would have resulted in an estimate of risk higher for the medium-exposure class than for the high-exposure class.

The reasons for this occurrence are threefold: (i) the nominal exposure range assigned to the high-exposure class ( $\geq 2 \mathrm{mG}$ ) was too high, that is, it was inappropriate to assign exposure scores equal to the exposure level ( 0 for low, 1 for medium, 2 for high) [this is a common method in exposure-response tests (15)]; (ii) misclassification affected different levels in different directions [while misclassification of the upper levels was severe, none of the subjects empirically classified into the category medium exposure were found to have truly low exposure]; (iii) the job description was not sufficiently rigorous. The most serious case of misclassification was due to the very high exposure $(49.5 \mathrm{mG})$ received by a mechanical workshop staff member during electric welding. This occurrence could have been prevented by a more precise definition of the job description (machine tool operators instead of mechanical workshop staff).

Some or all of these factors are likely to affect several of the exposure assessment surrogates commonly used in epidemiology, and therefore a pattern of misclassification similar to that described in this paper is probably not uncommon.

This phenomenon leads to the conclusion that, while the presence of a consistent exposure-response relationship may be used as grounds for inferring causality, its absence should not be construed as evidence of an artifactual association.

\section{References}

1. McDonald AD, Cherry NM, Delorme C, McDonald JC. Visual display units and pregnancy: evidence from the Montreal survey. J Occup Med 1986;28:1226-31.

2. Bryant HE, Lowe EJ. Video display terminal use and spontaneous abortion risk. Int J Epidemiol 1989;18: $132-38$.

3. Keam DW. Wire coding configurations are poor surrogates for magnetic field exposure. Radiat Prot Aust 1988;6:82-6.

4. Tomenius L, Hellstrom L, Enander B. Electrical constructions and $50 \mathrm{~Hz}$ magnetic fields at dwellings of tumor cases ( $0-18$ years of age) in the county of Stockholm. In: Proceedings of the international symposium on occupational health and safety in mining and tunnelling, Prague, June 21-25, 1982. Communication 71, 1-5 (microfiche).

5. Dosemici M, Wacholder S, Lubin JH. Does nondifferential misclassification of exposure always bias a true effect toward the null value? Am J Epidemiol 1990;132: 746-48.

6. Flegal KM, Brownie C, Haas JD. The effects of exposure misclassification on estimates of relative risk. Am J Epidemiol 1986;123:736-51.

7. Gladen B, Rogan WJ. Misclassification and the design of environmental studies. Am J Epidemiol 1979;109: 607-16.

8. Coleman M, Beral V. A review of epidemiological studies of the health effects of living near or working with electricity generation and transmission equipment. Int J Epidemiol 1988;17:1-13.

9. Delpizzo V. An evaluation of the existing evidence on the carcinogenic potential of extremely low frequency magnetic fields. Australas Phys Eng Sci Med 1989;12: $55-68$.

10. Dennis JA, Muirhead CR, Ennis JR. Epidemiological studies of exposure to electromagnetic fields: II. cancer. J Radiol Prot 1991;1:13-25.

11. Delpizzo V, Salzberg MR. Relative risk bias due to nondifferential misclassification of exposure in epidemiological studies using an ordinal exposure scale. Radiat Prot Australia 1990;4:91-4.

12. Delpizzo V, Salzberg MR. Relative risk estimate bias and loss of power in the Mantel test for trend resulting from the use of magnetic field "spot" measurements in epidemiological studies using an ordinal exposure scale. Bioelectromagnetics. In press.

13. Wacholder S, Dosemici M, Lubin JH, Blind assignment of exposure does not always prevent differential misclassification. Am J Epidemiol 1991;134:433-7.

14. Flegal KM, Keyl PM, Nieto FJ. Differential misclassification arising from non-differential errors in exposure measurement. Am J Epidemiol. 1991;134:1233-40.

15. Schlesselman JJ. Case-control studies. New York, NY: Oxford University Press, 1982.

Received for publication: 7 November 1991 